\title{
Search for high-energy neutrinos from GW170817 with the Baikal-GVD neutrino telescope
}

\author{
A.D. Avrorin ${ }^{1}$, A. V.Avrorin ${ }^{1}$, V. M. Aynutdinov ${ }^{1}$, R. Bannash ${ }^{7}$, I. A.Belolaptikov' ${ }^{2}$, V. M. Brudanin ${ }^{2}$, \\ N.M.Budnev ${ }^{3}$, A. A. Doroshenko ${ }^{1}$, G. V. Domogatsky ${ }^{1}$, R. Dvornickí2,8, A. N. Dyachok ${ }^{3}$, \\ Zh.-A.M.Dzhilkibaev ${ }^{1+1)}$, L. Fajt ${ }^{2,8,9}$, S. V.Fialkovsky ${ }^{5}$, A. R. Gafarov ${ }^{3}$, K. V. Golubkov ${ }^{1}$, T. I. Gres ${ }^{3}$, Z. Honz ${ }^{2}$,

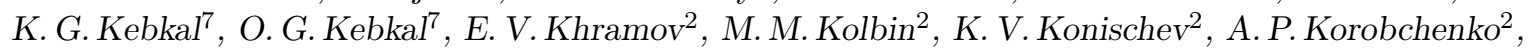 \\ A. P. Koshechkin ${ }^{1}$, V. A. Kozhin ${ }^{4}$, V.F.Kulepov ${ }^{5}$, D. A. Kuleshov ${ }^{1}$, M. B. Milenin ${ }^{5}$, R. A. Mirgazov ${ }^{3}$, E. R. Osipova ${ }^{4}$, \\ A. I. Panfilov' ${ }^{1}$ L. V.Pan'kov ${ }^{3}$, D. P.Petukhov ${ }^{1}$, E. N. Pliskovsky ${ }^{2}$, M. I. Rozanov ${ }^{6}$, E. V. Rjabov ${ }^{3}$, V. D. Rushay ${ }^{2}$, \\ G. B. Safronov ${ }^{2}$, F. Simkovic ${ }^{2,8}$, A. V.Skurikhin ${ }^{4}$, B. A.Shoibonov ${ }^{2}$, A. G. Solovjev ${ }^{2}$, M. N. Sorokovikov ${ }^{2}$, \\ M. D. Shelepov ${ }^{1}$, I. Shtekl ${ }^{2,9}$, O. V.Suvorova ${ }^{1}$, V. A. Tabolenko ${ }^{3}$, B. A. Tarashansky ${ }^{3}$, S. A. Yakovlev ${ }^{7}$, \\ A. V. Zagorodnikov ${ }^{3}$, V.L.Zurbanov ${ }^{3}$ \\ ${ }^{1}$ Institute for Nuclear Research RAS, 117312 Moscow, Russia \\ ${ }^{2}$ Joint INstitute for Nuclear Research, 141980 Dubna, Russia \\ ${ }^{3}$ Irkutsk State University, 664003 Irkutsk, Russia \\ ${ }^{4}$ Institute of Nuclear Physics, Moscow State University, 119991 Moscow, Russia \\ ${ }^{5}$ Nizhni Novgorod State Technical University, 603950 Nizhni Novgorod, Russia \\ ${ }^{6}$ St. Petersburg State Marine Technical University, 190008 St. Petersburg, Russia \\ ${ }^{7}$ EvoLogics, 13355 Berlin, Germany \\ ${ }^{8}$ Comenius University, 84248 Bratislava, Slovakia \\ ${ }^{9}$ Czech Technical University, 12800 Prague, Czech Republic \\ Submitted 24 October 2018
}

\begin{abstract}
The Advanced LIGO and Advanced Virgo observatories recently discovered gravitational waves from a binary neutron star inspiral. A short gamma-ray burst (GRB) that followed the merger of this binary was also recorded by Fermi-GBM and INTEGRAL, indicating particle acceleration by the source. The precise location of the event was determined by optical detections of emission following the merger. We searched for high-energy neutrinos from the merger in the TeV - $100 \mathrm{PeV}$ energy range using Baikal-GVD. No neutrinos directionally coincident with the source were detected within $\pm 500 \mathrm{~s}$ around the merger time, as well as during a 14-day period after the GW detection. We derived $90 \%$ confidence level upper limits on the neutrino fluence from GW170817 during a $\pm 500 \mathrm{~s}$ window centered on the GW trigger time, and a 14-day window following the GW signal under the assumption of an $E^{-2}$ neutrino energy spectrum.
\end{abstract}

Introduction. A gravitational wave signal, GW170817, from a binary neutron star merger has been recorded by the Advanced LIGO and Advanced Virgo observatories on August 17, 2017 1]. A short GRB (GRB170817A), associated with GW170817, was detected by Fermi-GBM and INTEGRAL. NGC 4993 was localized as the host galaxy of the merger by follow up optical observations. High-energy neutrino signals associated with the merger were searched for by the ANTARES and IceCube neutrino telescopes in muon and cascade modes and the Pierre Auger Observatory 2, and Super-Kamiokande [3]. Two

\footnotetext{
1) e-mail: djilkib@yandex.ru
}

different time windows were used for the searches. First, a $\pm 500 \mathrm{~s}$ time window around the merger was used to search for neutrinos associated with prompt and extended gamma-ray emission [4, 5]. Second, a 14-day time window following the GW detection, to cover predictions of longer-lived emission processes [6. 7]. No significant neutrino signal was observed by the neutrino telescopes.

The deep underwater neutrino telescope Baikal Gigaton Volume Detector (Baikal-GVD) is currently under construction in Lake Baikal [8]. The telescope has a modular structure and consists of functionally independent sub-arrays (clusters) of optical modules 


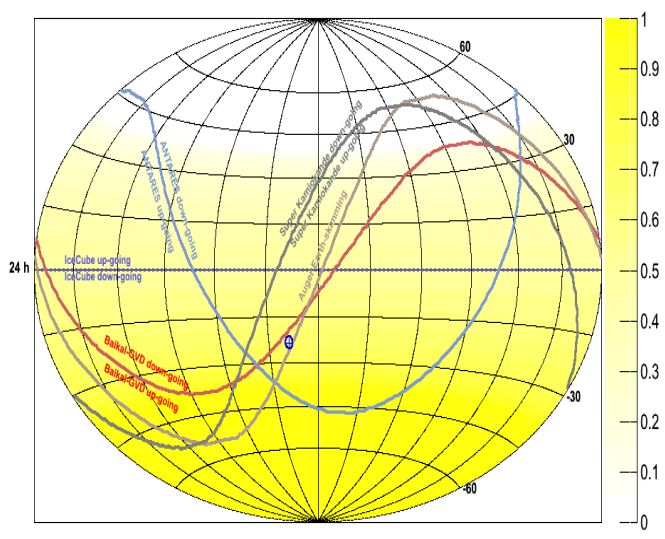

Figure 1. Localizations of NGC 4993 and horizons separating down-going and up-going neutrino directions for IceCube, ANTARES, SuperKamiokande and BaikalGVD at the time of the GW event in equatorial coordinates. The zenith angle of the source at the detection time of the merger was $73.8^{\circ}$ for ANTARES, $66.6^{\circ}$ for IceCube, $108^{\circ}$ for SK and $93.3^{\circ}$ for BaikalGVD.

(OMs). Since each GVD-cluster represents a multimegaton scale Cherenkov detector, studies of neutrinos of different origin are allowed at early stages of construction. During 2017 two GVD-clusters have been operated in Lake Baikal. In this Letter we present searches for high-energy neutrinos in coincidence with GW170817/GRB170817A by the Baikal-GVD highenergy neutrino telescope.

Detector and search method. The geographical coordinates of the Baikal-GVD site are $51^{\circ} 50^{\prime} \mathrm{N}$ and $104^{\circ} 20^{\prime} \mathrm{E}$. The detector instruments the deep water of Lake Baikal with optical modules - pressure resistant glass spheres equipped with photomultiplier tubes (PMT) Hamamatsu R7081-100 with photocathode diameter of 10 " and a quantum efficiency of $\sim 35 \%$ [9]. The PMTs record the Cherenkov radiation from secondary particles produced in interactions of highenergy neutrinos inside or near the instrumented volume. From the arrival times of light at the PMTs and from the amount of light, direction and energy of the incoming neutrinos are derived. Baikal-GVD in it's 2017 design consisted of two clusters - each of them with 288 optical modules. A cluster comprises eight vertical strings attached to the lake floor: seven side strings on a radius of $60 \mathrm{~m}$ around a central one. Each string carries $36 \mathrm{OMs}$, arranged at depths between 735 and 1260 meters (525 m instrumented length). The vertical spacing between the OMs along a string is 15 $\mathrm{m}$. The OMs on each string are functionally combined in 3 sections. A section comprises $12 \mathrm{OMs}$ with data processing and communication electronics and forms a detection unit (DU) of the array. All analogue signals from the PMTs are digitized, processed in the sections and sent to shore if certain trigger conditions (e.g. a minimum number of fired PMTs) are fulfiled [10].

IceCube discovered a diffuse flux of high-energy astrophysical neutrinos in 2013 [11. The data sample of their high-energy starting event analysis (HESE, 7.5 year sample) comprises 103 events, 77 of which are identified as cascades and 26 as track events [12]. These results demonstrate the importance of the cascade mode of neutrino detection with neutrino telescopes. A search for high-energy neutrinos associated with GW170817 in Baika-GVD is based on the selection of cascade events generated by neutrino interactions in the sensitive volume of array. The procedure for reconstructing the parameters of high-energy showers the shower energy, direction, and vertex - is performed in two steps. In the first step, the shower vertex coordinates are reconstructed by $\chi_{t}^{2}$ minimization using the time information from the telescope's triggered photo-sensors. The reconstruction quality can be increased by applying additional event selection criteria based on the limitation of the admissible values for the specially chosen parameters characterizing the events. In the second step, the shower energy and direction are reconstructed by applying the maximum-likelihood and using the shower coordinates reconstructed in the first step. The values of the variables $\theta, \phi$, and $\mathrm{E}_{s h}$ corresponding to the maximum value of the likelihood are chosen as the polar and azimuth angles characterizing the direction and the shower energy.

The zenith angle of NGC 4993 at the detection time of GW170817 was 93.3 ${ }^{\circ}$ for Baikal-GVD (see, Figure 11. Since background events from atmospheric muons and neutrinos can be significantly suppressed by requiring time and space coincidence with the GW signal, relatively weak cuts can be used for neutrino selection. For the search for neutrino events within a $\pm 500 \mathrm{~s}$ window around the GW event, 731 events were selected, which comprise $>5$ hit OMs at $>2$ hit strings. After applying cascade reconstruction procedures and dedicated quality cuts, two events were selected. Finally, requiring directional coincidence with NGC $4993 \psi<$ $20^{\circ}$ no neutrino candidates survived. Shown in Figure 2 are temporal distributions of events fulfilling the initial selection requirement as well as events surviving all cuts during the $39347 \mathrm{~s}$ long data taking run, which contains the $\pm 500 \mathrm{~s}$ time window around GW170817. Neutrino 


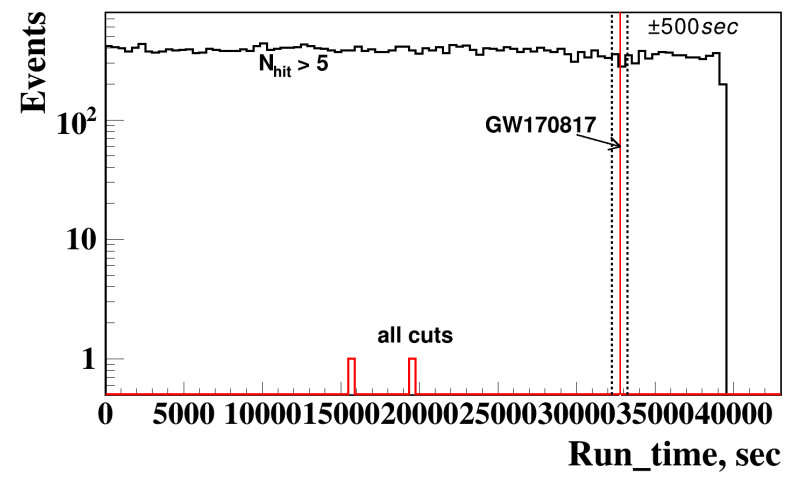

Figure 2. Temporal distribution of events during the data taking run contaning the $\pm 500 \mathrm{~s}$ time window around the GW event. The histograms represent events with hit $\mathrm{OMs}_{\text {hit }}>5$, and events surviving all selection cuts used for the neutrino search within $\pm 500 \mathrm{~s}$ time window around the GW event.
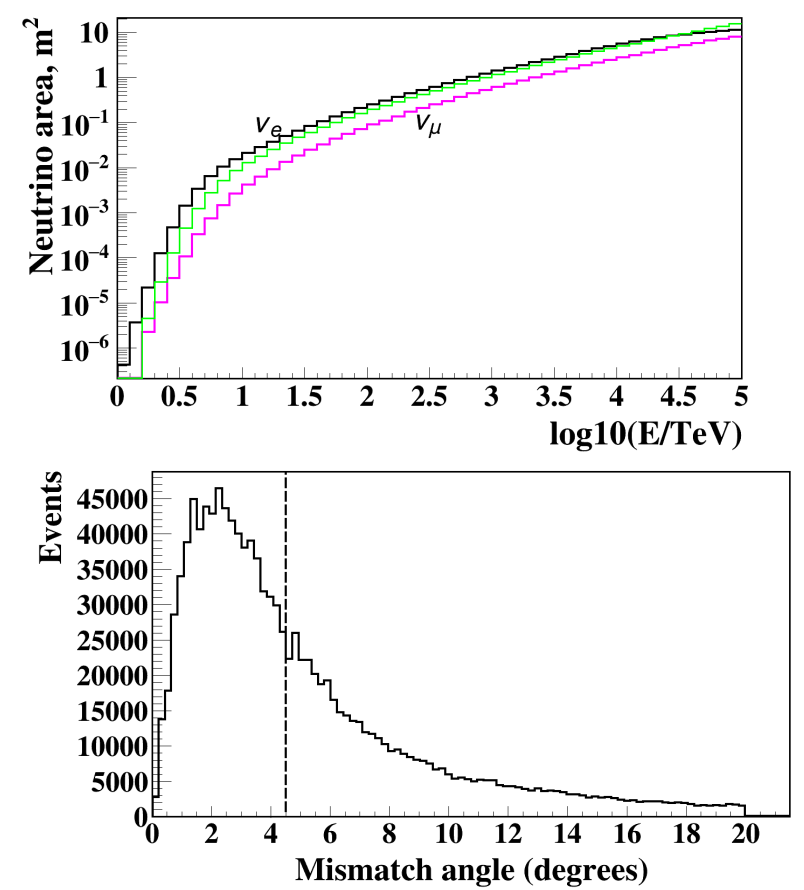

Figure 3. Neutrino effective areas (top panel) and error angles distribution (bottom panel).

effective areas for each flavour are shown in Figure 3 (top panel). Error angles distribution is shown in Figure 3 (bottom panel). The median angular error is $4.5^{\circ}$ with this set of relaxed cuts and the expected number of atmospheric background events is about $5 \times 10^{-2}$ during the coincident time window.

The absence of neutrino candidates in the $\pm 500 \mathrm{~s}$ window associated with GW170817 allows to constrain the fluence of neutrinos from GW170817A. Assuming

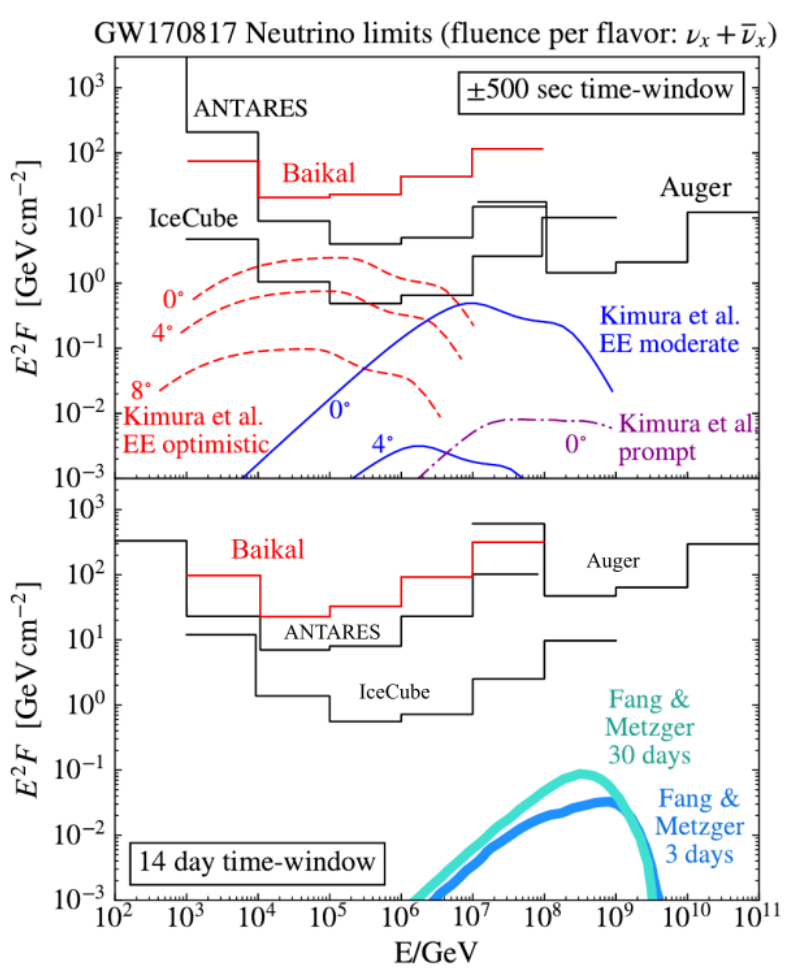

Figure 4. Upper limits (at $90 \%$ confidence level) on the neutrino spectral fluence from GW170817 during a $\pm 500 \mathrm{~s}$ window centered on the GW trigger time (top panel), and a 14-day window following the GW trigger (bottom panel). For each experiment, limits are calculated separately for each energy decade, assuming a spectral fluence $F(E)=F_{u p} \times[E / G e V]^{-2}$ in that decade only. Also shown are predictions by neutrino emission models (see [2] for details).

an $E^{-2}$ spectrum single-flavor differential limits to the spectral fluence in bins of one decade in energy have been derived according to 13. (see Figure 4(top panel)). In the range from $5 \mathrm{TeV}$ to $10 \mathrm{PeV}$ a $90 \% \mathrm{CL}$ upper limit on an $E^{-2}$ power-law spectral neutrino fluence is $5.2 \times(E / G e V)^{-2} \mathrm{GeV}^{-1} \mathrm{~cm}^{-2}$.

The search over 14 days used a more stringent cut on the number of hit OMs $-\mathrm{N}_{\text {hit }}>7$. The zenith angle of the optical counterpart oscillates daily between $74^{\circ}$ and $150^{\circ}$. No events spatially coincident with GRB170817A were found in this search. Given the non-detection of neutrino events associated with GW170817, differential upper limits have been derived (see Figure 4 (bottom panel)). The corresponding upper limit to the spectral fluence is $9.0 \times(E / G e V)^{-2} \mathrm{GeV}^{-1} \mathrm{~cm}^{-2}$ over the same energy range as for the $\pm 500 \mathrm{~s}$ time window.

Conclusion. The deep underwater neutrino telescope Baikal-GVD is currently under construction. During 2017 two GVD-clusters have been operated in Lake Baikal. We performed a search for high-energy 
neutrinos associated with the gravitational wave, GW170817, from the binary neutron star merger in NGS4993 with Baikal-GVD, using the cascade detection mode. The search was performed within a $\pm 500 \mathrm{~s}$ time window around GW170817, and 14 days after detection of the gravitational wave. No neutrino events associated with the merger were detected within the $\pm 500 \mathrm{~s}$ time interval, nor during 14 days after the gravitational wave. This allows to derive the neutrino spectral fluence limits from this merger. The ongoing analysis of GVD data using the muon detection mode will allow to improve these limits.

This work was supported by the Russian Foundation for Basic Research (Grants 16-29-13032, 17-02-01237).

1. B. Abbott et al., Phys. Rev. Lett., 119, 161101 (2017).

2. A. Albert et al., arXiv:1710.05839, (2017).

3. K. Abe, B., et al., arXiv:1802.04379, (2018).

4. B. Baret et al., Astropart. Phys. 35, 1 (2011).

5. S. Kimura et al., ApJL 848, L4 (2017).

6. H. Gao et al., , Phys. Rev. D88, 043010 (2013).

7. K. Fang and B. Metzger, arXiv:1707.04263, (2017).

8. A.Avrorin et.al., PoS (ICRC2017)1034, (2017)

9. A.D. Avrorin et al., EPJ Web Conf. 116, 01003 (2016).

10. A.D. Avrorin et al., Instr.Exper.Tech. 57, 262 (2014).

11. M. G. Aartsen et al., IceCube Coll., Science, 342, 1242856 (2013), arXiv:1311.5238 [astro-ph.HE]].

12. I. Taboada, Talk at XXVIII International Conference on Neutrino Physics and Astrophysics, 4-9 June 2018, Heidelberg, Germany, DOI: 10.5281/zenodo.1286918, URL: https://doi.org/10.5281/zenodo.1286918.

13. G. Feldman and R. Cousins, Phys. Rev. D 57, 3873 (1998). 